\title{
Calcul numérique de certains écoulements par la théorie des tourbillons
}

\author{
par R. Gelci \\ Météorologie Nationale
}

\section{Introduction}

La théorie des tourbillons, élaborée voici plus de cent ans, se prête à des applications directes en hydrodynamique plane.

Elle permet en particulier de calculer simplement l'évolution d'un écoulement à potentiel, engendré par des répartitions tourbillonnaires curvilignes, sans qu'il soit nécessaire d'expliciter le potentiel.

Tel est par exemple le cas d'écoulements en présence d'obstacles, sans décollement; la vitesse de glissement, équivalente à un tourbillon (lié) est solution d'une équation intégrale de Fredholm de 2ème espèce. La circulation, qui se conserve, constitue une condition initiale.

Tel est aussi le cas des mouvements irrotationnels avec gravité, comportant une ou plusieurs lignes de discontinuité de vitesse et de densité (surface libre). Dans ce cas, les positions successives des tourbillons libres sont calculées par la relation de Poincaré, ef leur intensité par une relation où intervient essentiellement la composante tangentielle de la pesanteur.

Tel est enfin le cas d'écoulement potentiel en présence d'obstacles anguleux avec décollement.

Dans ce cas, la circulation autour de l'obstacle ne se conserve pas, mais diminue algébriquement de la quantité de tourbillons libérée en chaque point anguleux selon l'une des deux tangentes.

\section{Vitesse induite par une ligne de tourbillons}

Si le point $M$ est extérieur à la ligne tourbillonnaire $A B$, la relation de Poincaré s'écrit:

$$
\vec{V}_{M}=\frac{1}{2 \pi} \int_{A}^{B} \frac{G(s) d s}{r^{2}}(\vec{k} \Lambda \vec{r})
$$

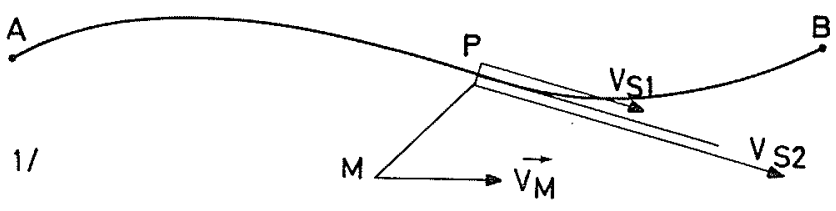

avec $G(s)=V_{S 2}-V_{S 1}$ où $V_{S 2}(s)$ et $V_{S 1}(s)$ sont les vitesses tangentielles en $P$ de part et d'autre de la ligne, à l'abscisse curviligne $s=A P$.

On a nécessairement: $G_{A}=G_{B}=0$

$\vec{k}$ est le vecteur unitaire perpendiculaire au plan du mouvement, et $\vec{r}=P \vec{M}$

Dans le cas où $M$ est situé sur la ligne tourbillonnaire, l'intégrale (1) n'est pas absolument convergente. 
Elle cunserve toutefois un sens.

Les composantes tangentielle et normale s'écrivent:

$$
\begin{aligned}
& V_{S}(s)=\frac{1}{2 \pi} \int_{A}^{B} g(\sigma, s) G(\sigma) d \sigma \\
& V_{N}(s)=\frac{1}{2 \pi} \int_{A}^{B} h(\sigma, s) G(\sigma) d \sigma
\end{aligned}
$$

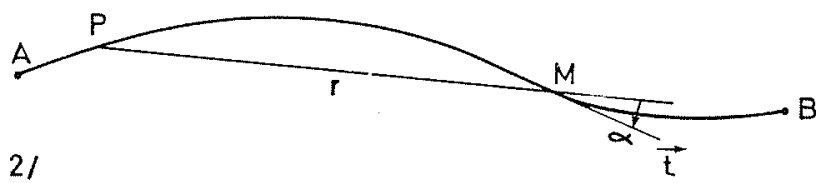

$$
\begin{aligned}
& \text { où } g(\sigma, \mathrm{s})=\frac{\sin a}{r} \quad h(\sigma, s)=\frac{\cos a}{r} \\
& \text { avec } a=(P \vec{M}, \vec{t})
\end{aligned}
$$

$\sigma$ étant l'abscisse curviligne du point courant $P$,

$s$ étant l'abscisse curviligne de $M$.

Le noyau de (2) reste fini lors que $P$ tend vers $M$.

On a en effet $\frac{\sin \alpha}{r} \rightarrow \frac{1}{2 R}$ lorsque $\sigma \rightarrow s$

$R$ étant le rayon de courbure en $M(s)$

On a enfin $V_{S}=\frac{1}{2}\left(V_{S 1}+V_{S 2}\right)$

$$
\begin{aligned}
& v_{S 1}=v_{S}+\frac{1}{2} G \\
& v_{S 2}=v_{s}-\frac{1}{2} G
\end{aligned}
$$

\section{Diffraction par un contour fermé sans décollement}

Dans le cas d'un obstacle régulier, soumis à l'écoule. ment incident $\vec{k}$, la vitesse $V_{S 1}$ est nulle et $G \equiv V_{S 2}$.

Les relations (2) et (3) deviennent alors, en tenant compte de l'écoulement incident, ef en faisant abstraction de l'indice 2 :

$$
\begin{aligned}
& \frac{1}{2} \mathrm{~V}_{\mathrm{S}}(\mathrm{s})=\frac{1}{2 \pi} \oint \mathrm{g}(\sigma, \mathrm{s}) \cdot \mathrm{V}_{\mathrm{S}}(\sigma) \mathrm{d} \sigma+\mathrm{K}_{\mathrm{S}}(\mathrm{s}) \\
& \mathrm{V}_{\mathrm{N}}(\mathrm{s})=\frac{1}{2 \pi} \oint \mathrm{h}(\sigma, \mathrm{s}) \cdot \mathrm{V}_{\mathrm{S}}(\sigma) \mathrm{d} \sigma+\mathrm{K}_{\mathrm{N}}(\mathrm{s})
\end{aligned}
$$

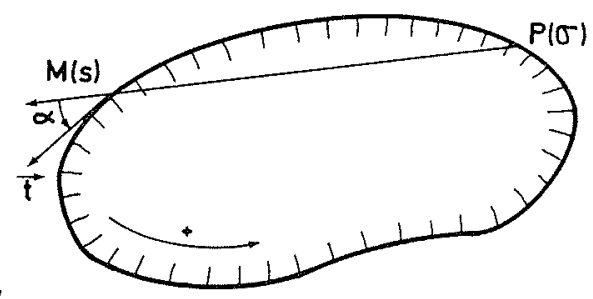

$K_{S}(s)$ et $K_{N}(s)$ étant les composontes tangentielle et normale en $M(s)$ de l'écoulement incident $\vec{K}$ (fig. 3)

Le calcul numérique de la vitesse de glissement $V_{S}$ peut être effectué à partir de (7) où l'on fait

$$
V_{N}(s)=0
$$

On est alors amené à résoudre une équation intégrale de Fredholm de 2ème espèce dont le noyau $h(\sigma, s)$ devient infini lorsque $\sigma \rightarrow \mathrm{s}$.

Des modèles, élaborés sur ce principe $[1,2]$, ont conduit à des résultats satisfaisants.

En réalité, la relation (6), qui se présente sous la forme d'une intégrale de Fredholm de 2 ème espèce, dont le noyau reste fini, convient mieux au calcul de la vitesse de glissement $V_{S}(s)$.

Elle fournit ai sément la solution dans le cas d'un contour circulaire de rayon $\mathrm{R}$.

On a alors: $g(\sigma, s)=\frac{1}{2 R}$ et

$$
\begin{gathered}
\frac{1}{2} V_{S}(s)=\frac{1}{2 \pi} \cdot \frac{1}{2 R} \oint V_{S}(\sigma) d \sigma+K_{S}(s) \\
V_{S}(s)=\frac{\Gamma}{2 \pi R}+2 K_{S}(s)
\end{gathered}
$$

soit

$\Gamma$ étant la circulation.

Dans le cas d'un contour quelconque, mais cependant pas trop allongé ou trop concove(6), peut être résolu par itérations.

Il est facile de montrer que la circulation se conserve au cours des itérations. C'est pourquoi l'initialisation $V_{5}^{o}(s)$ doit correspondre à la circulation $\Gamma$, définie comme condition initiale.

Un modèle de diffraction a été réalisé [6] en discrétisant le contour par $\mathrm{N}-\mathrm{I}$ arcs de courbes analytiques, de manière à assurer la continuité de la tangente et du rayon de courbure en chaque point de discrétisation. 
$V_{S}(s)$ est approximé, sur l'arc $(1,1+1)$ par une forme linéaire de $V_{\mathrm{SI}-1}, V_{\mathrm{SI}}, \mathrm{V}_{\mathrm{SI}+1}, \mathrm{~V}_{\mathrm{SI}+2} \cdot(6)$ se présente alors sous la forme:

$$
\left[\begin{array}{c}
v_{S 1} \\
v_{S 2} \\
\\
v_{S N}
\end{array}\right]
$$$$
\left[\begin{array}{c}
v_{S 1} \\
v_{S 2} \\
\\
v_{S N}
\end{array}\right]
$$$$
\left[\begin{array}{c}
K_{S 1} \\
K_{S 2} \\
\\
K_{S N}
\end{array}\right]
$$

\|A $\mid$ étant une matrice $(N, N)$ ne dépendant que de l'obstacle et de la forme linéaire adoptée pour une interpolation de $V_{S}$.

Les résultats du modèle numérique DTX3 ainsi réalisé sont satisfaisants.

On trouvero, sur les planches 1 à 4 les vitesses de glissement ainsi obtenues pour divers types de contour.

Dans le cas de contours présentant des allongements ou des concavités trop accusés, la convergence des itérations n'est plus assurée; mais il est en général facile de diminuer cet allongement ou cette concavité par une transformation conforme intermédiaire relativement simple.

\section{Diffraction sans décollement par un contour périodique}

Dans le cas d'un tel contour, la relation (2) s'écrit:

$$
\frac{1}{2} V_{S}(s)=\frac{1}{2 \pi} \int_{-\infty}^{+\infty} g(\sigma, s) V_{S}(\sigma) d \sigma+K_{S}(s)
$$

L'intégrale peut se ramener à la somme de $N$ séries absolument convergentes, $N$ étant le nombre de points de discrétisation d'une longueur du contour.

Le modèle DTXP, réalisé sur ce principe $[7,8]$ permet ainsi de calculer la vitesse de glissement d'un courant le long d'un contour périodique.

Les planches 5 et 6 représentent ces vitesses de glis. sements le long de profils sinusoidaux et eycloidaux de différentes cambrures.

\section{Évolution d'une ligne de discontinuité de vitesse}

Etant donné une ligne $A B$ de discontinuité de vitesse, la relation (1) permet de calculer la vitesse $\vec{V}_{M}$ d'un point quel conque $\mathrm{M}$ de $\mathrm{AB}$.
Au cours de l'évolution, le tourbillon $G(s)$ ds se conserve, soit : $\quad \frac{D}{D r}[G(s) d s]=0$

$\frac{D}{D t}$ étant un opérateur lié à $\vec{V}_{M}$

On a donc, en posant $\delta(s)=\frac{1}{d s} \frac{D}{D t}(d s)$

$$
\frac{D}{D t}[G(s)]=-\delta(s)
$$

$\delta(s)$, comme $\vec{V}_{M}(s)$ ne dépend que de la discontinuité $G(s)$, et de sa géométrie.

L'évolution de $G(s)$ est done connue à chaque instant.

Sur ce principe ont été élaborés plusieurs modèles numériques $[3,4,5]$. Les planches $7,8,9$ représentent l'évolution d'un certain nombre de lignes de discontinuité, avec, au regard, la valeur initiale de $G(s)$.

\section{Évolution d'une surface libre}

Dans ce cas, la vitesse $\vec{V}_{M}$ d'un point $M$ quelconque de la "surface libre" est encore foumie par la relation de Poincaré (1).

Mais l'évolution de la discontinuité de vitesse devient $[3,9]$

$$
\frac{1}{d s} \frac{D}{D t}[G(s) d s]=\frac{\rho_{2}-\rho_{1}}{\frac{\rho_{1}+\rho_{2}}{2}}\left[g_{S}-\frac{G}{4} \frac{\partial G}{\partial s}-J_{S}\right]
$$

$\rho_{1}$ et $\rho_{2}$ étant les masses spécifiques respectives des liquides supérieur et inférieur,

$J_{S}$ étant l'accélération tangentielle d'un point $M$ de la surface libre de vitesse $\vec{V}_{M}$

${ }^{9}$ étant la composante tangentielle de la gravité le long de la surface libre.

La relation (9) apparaît comme un cas particulier de (11) lorsque $\rho_{1}=\rho_{2}$.

L'accélération tangentielle $J_{S}$ peut être exprimée à partir d'intégrales curvilignes portant sur $G(s)$ ef $D[G(s)] / D t$, en sorte que (11) se présente sous la forme d'une équation intégrale de Fredholm de 2ème espèce. 
En pratique $J_{S}$ est négligeable devant $g_{S^{\prime}}$ ainsi d'ailleurs que $G \frac{\partial G}{\partial s} / 4$, en sorte que (11) se réduit à la relation d'évolution

$$
\frac{D}{D+}[G(s) d s]=\frac{\rho_{2}-\rho_{1}}{\frac{\rho_{1}+\rho_{2}}{2}} g_{S}
$$

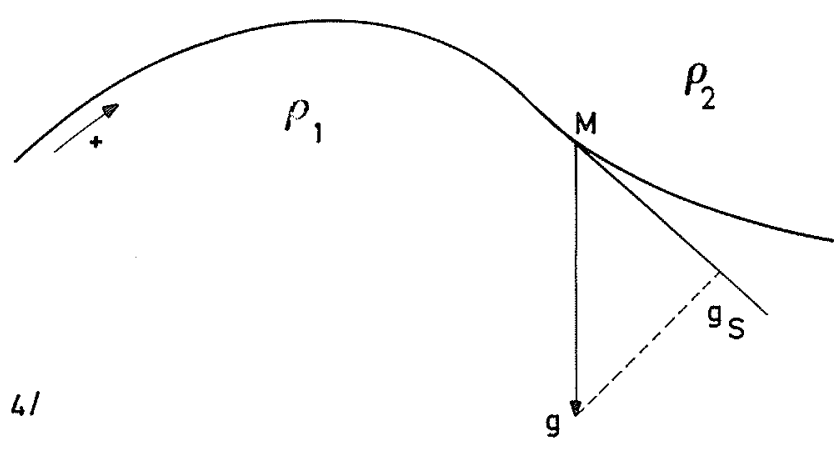

Un modèle numérique a été élaboré $[8,9]$ à partir des relations (1) et (12).

La surface libre est supposée périodique.

Dans le cas particulier où celle-ci est, initialement, sinusoidale, et de cambrure inférieure à $5 \%$, le modèle en assure une remarquable conservation.

Dans le cas de fortes cambrures (supérieures à $5 \%$ ), il apparaît un déferlement local au voisinage de la crête, au bout d'un temps d'autant plus court que la cambrure est plus forte.

Le modèle permet, entre autres, la mise en évidence du courant d'entraínement des particules fluides supérieures ef inférieures, avec une très bonne précision.

\section{Diffraction avec sillage}

Considérons un contour orienté présentant un point anguleux $A$ d'abscisse curviligne $a$, de tangentes $\vec{t}$ ef $\vec{f}^{\prime}$ (fig. 5).

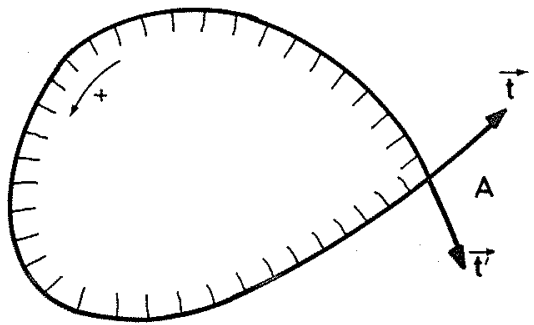

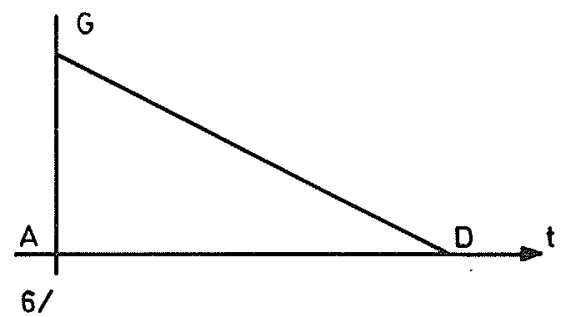

Du fait même de l'existence de ce point anguleux, il $n$ 'existe pas de vitesse $V_{S}(s)$ correspondant à l'écoulement incident $\vec{K}$.

Imaginons alors, ajoutée à l'écoulement incident $\vec{k}$, une discontinuité de vitesse $G(x)$ le long de la tangente positive $\vec{t}$, répartie sur cette tangente entre $A$ et un point voisin $D$ (fig. 6).

Il est alors possible de trouver une vitesse de glissement $V_{S}(s)$ discontinue en $A$, telle que :

$$
\frac{1}{2} V_{S}(s)=\frac{1}{2 \pi} \oint g(\sigma, s) V_{S}(\sigma) d \sigma+K_{S}(s)+D_{S}(s)
$$

$D_{S}(s)$ étant la composante tangentielle de la vitesse induite en $M(s)$ par la répartition tourbillonnaire $G(x)$ sur $A D$.

$$
\begin{aligned}
& \text { On a nécessairement } V_{S}(a+O)=0 \\
& \text { et } V_{S}(a-O)=G(O)>0
\end{aligned}
$$

Dans le cas où cette dernière condition n'est pas satisfaite, on imaginera une discontinuité de vitesse $G^{\prime}(x)$ le long de la tangente négative $\vec{t}^{\prime}$, répartie entre $A$ et un point voisin $D^{\prime}$ de cette tangente. La vitesse de glissement satisfera alors à la relation:

$$
\frac{1}{2} V_{S}(s)=\frac{1}{2 \pi} \oint g^{\prime}(\sigma, s) V_{S}(\sigma) d \sigma+K_{S}^{\prime}(s)+D_{S}^{\prime}(s)
$$

$D_{S}^{\prime}(s)$ étant la composante tangentielle de la vitesse induite en $M(s)$ par la répartition tourbillonnaire $G^{\prime}(x)$ sur $A D^{\prime}$.

D'autre part, $K_{S}^{\prime}(s) \equiv K_{S}(s)$ et $g^{\prime}(\sigma, s) \equiv g(\sigma, s)$ pour $s \neq a$.

On aura alors nécessairement:

$V_{S}(a-0)=0 \quad$ et $\quad V_{S}(a+0)=G^{\prime}(0)<0$

Comme pour un obstacle "arrondi", la circulation initiale de la vitesse de glissement constitue une condition ini- 
tiale du problème. Mais, dans le cas présent, cette circu. lation évolue selon la relation:

$$
\begin{aligned}
& d \Gamma+\left|\frac{1}{2} V_{S A} d t\right| V_{S A}=0 \\
& \frac{d \Gamma}{d t}=-\frac{1}{2}\left|V_{S A}\right| V_{S A}
\end{aligned}
$$

Cette variation de $\Gamma$ tend à déplacer progressivement vers A l'un des deux points du contour où la vitesse de glissement est nulle.

Parallèlement, le sillage initial évolue, conformément aux relations (1) et (10).

Un modèle numérique simplifié [10] a été élaboré pour un segment rectiligne $A B$. Les deux sillages sont discrétisés par des tourbillons ponctuels prenant naissance en $A^{\prime}$ et $B^{\prime}$, voisins des extrémités $A$ et $B$ du segment $A B$, et calculés de manière à annuler les vitesses $V_{A}$ et $V_{B}$.

L'allure générale des 2 sillages apparaît réaliste (figure 7) mais la force exercée trop faible (dans le rapport de 1 à 10).

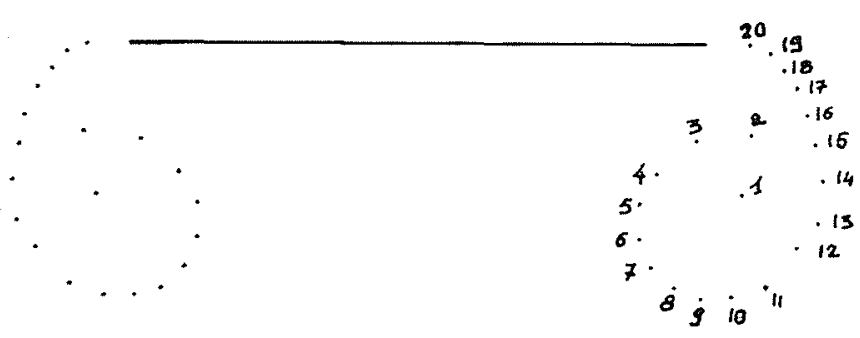

\section{Conclusions}

La relation de Poincaré se prête très bien au calcul numérique dans le cas de répartitions tourbillonnaires curvilignes $G(s)$, c'est-à-dire de lignes de discontinuité de vitesse.

Dans le cas de mouvements tourbillonnaires (rot $\vec{V}=\vec{k} v^{2} \psi$ ) la méthode ne semble pas numériquement, et a priori, supérieure à celle de la recherche directe de la fonction de courant $\psi$.
La méthode semble se prêter à la simulation de la croissance et de l'évolution des vagues ef des ondes internes dans des conditions assez réalistes.

En ce qui concerne les modèles d'interaction entre un écoulement et un obstacle décrits plus haut, la prise en compte de la couche limite semble devoir offrir quelques difficultés.

\section{Références}

[1] GELCI (R.) et LACROUTE (B.). - Diffraction d'un écoulement autour d'un obstacle par des tourbillons liés. Note de l'EERM n 261 - Janvier 1969.

[2] CLEMENT (B.). - Aspects numériques de la théorie des tourbillons, cas des mouvements plans. Thèse de Doctorat. Université de Nancy - Juillet 1969.

[3] GELCl (R.) et SCHAEFFER (A.). - Evolution d'une discontinuité de vitesse - Note de l'EERM $n^{\circ} 277$. Septembre 1970.

[4] SAIAC (J.H.). - Modèle de calcul numérique de l'évolution d'une ligne de discontinuité de vitesse - Note de I'EERM no 285 - Novembre 1971.

[5] SAIAC (J.H.) et BARY (R.). - Modèle d'évolution d'une ligne de discontinuité de vitesse - Note de l'EERM no 294 - Novembre 1971.

[6] GELCl (R.) et AMIEL (G.). - Calculs de diffraction plane - Note de I'EERM no 298 - Juin 1972.

[7] VAUTRAVERS (J.). - Ecoulements incompressibles le long d'un obstacle ou d'un interface périodiques - Note de l'EERM no 308 - Août 1972.

[8] VAUTRAVERS (J.). - Quelques applications numériques de la théorie des tourbillons à l'hydrodynamique des écoulements incompressibles. LA METEOROLOGIE $n^{0} 19$ - Juillet - Septembre 1971.

[9] VAUTRAVERS (J.). - Evolution d'une surface libre périodique au moyen de la théorie des tourbillons. Note de l'EERM no 327 - Juin 1973.

[10]RONGIER (J.). - Modèle numérique d'interaction entre un écoulement plan et un segment rectiligne. Compte rendu intérieur de l'EERM - Juin 1973.

(Toutes ces études ont été effectuées avec l'aide de la Direction des Recherches et Moyens d'Essais.) 

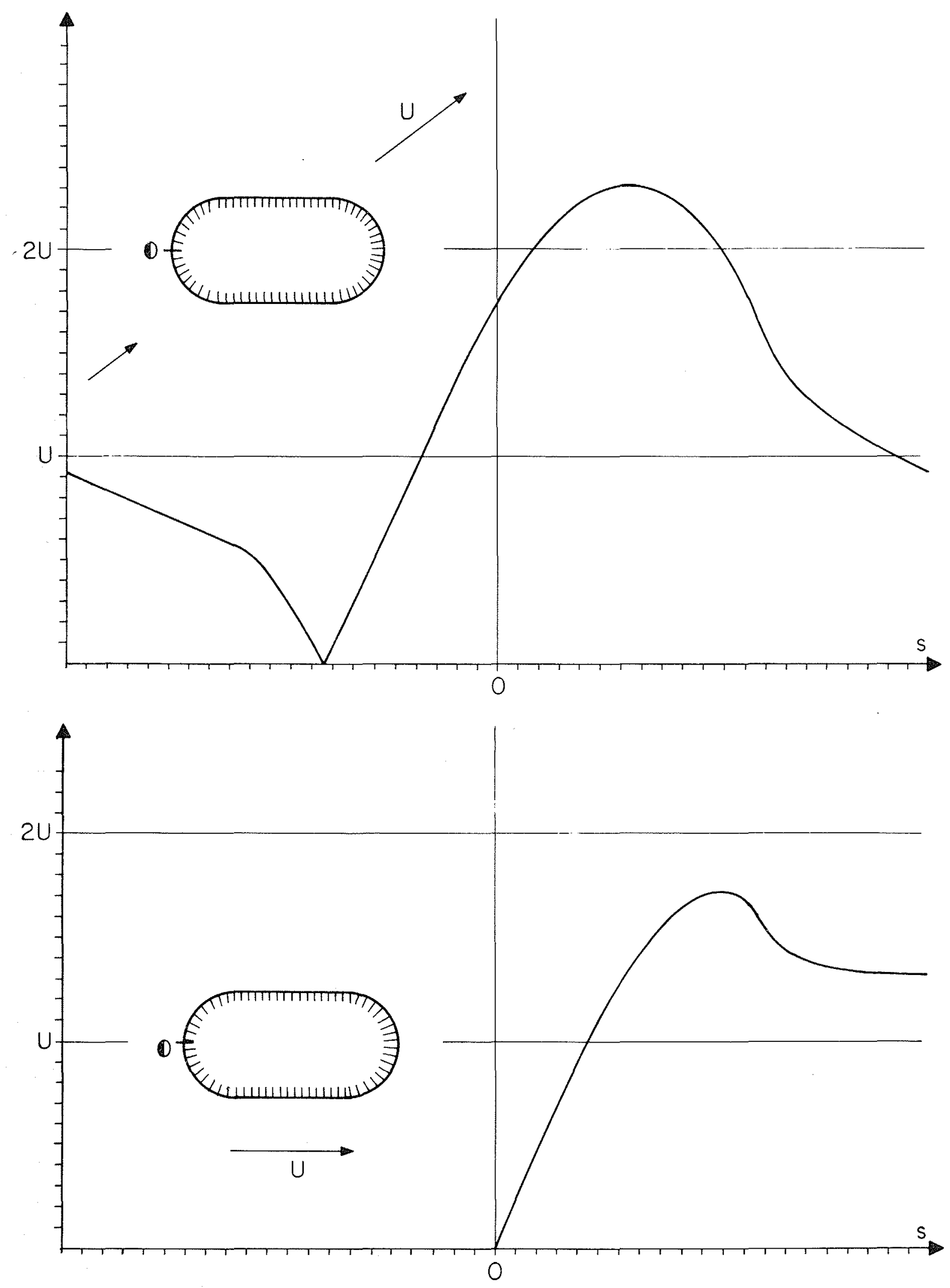

Planche 1/ 
R. GELCI
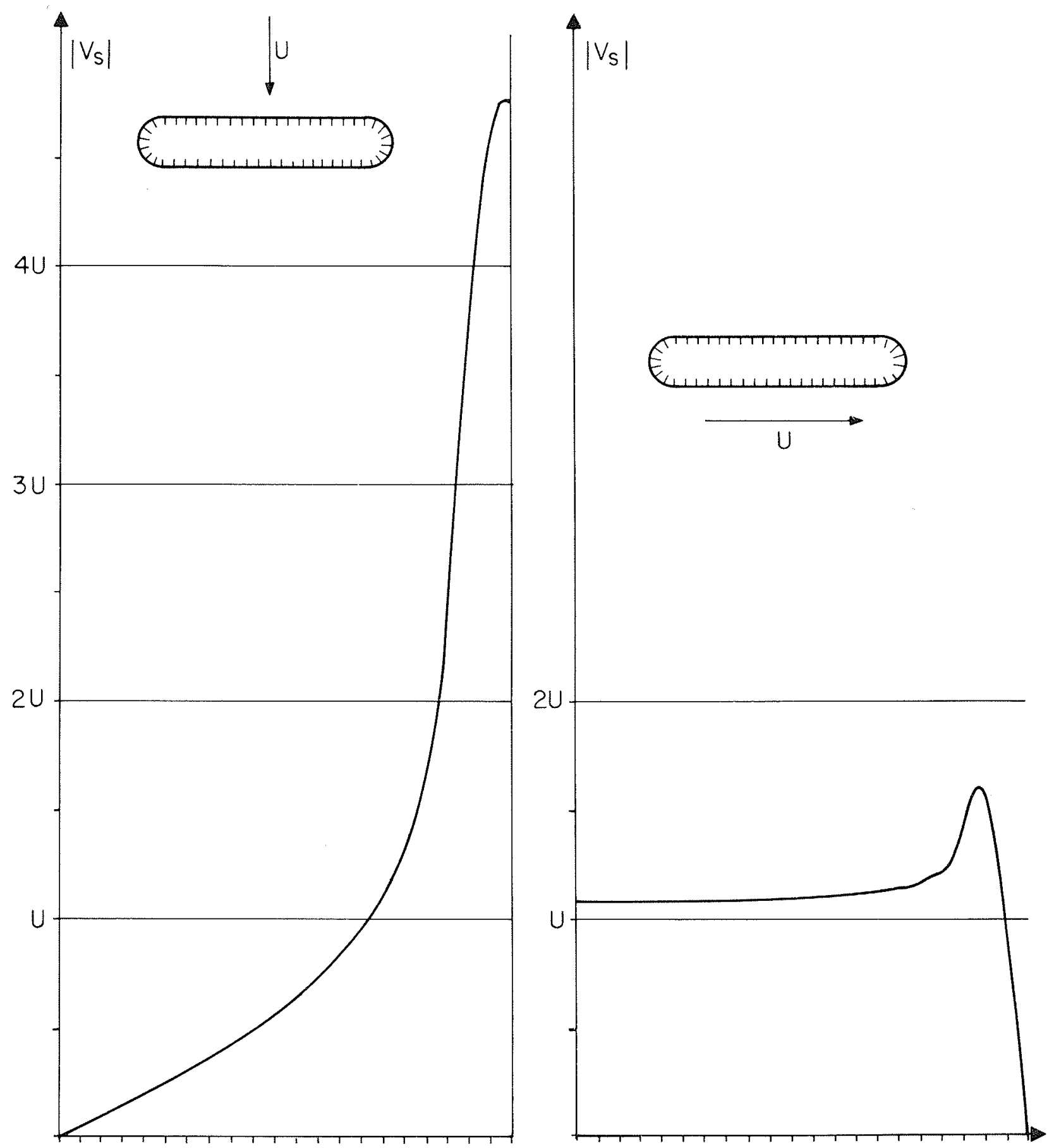

Planche 2/ 


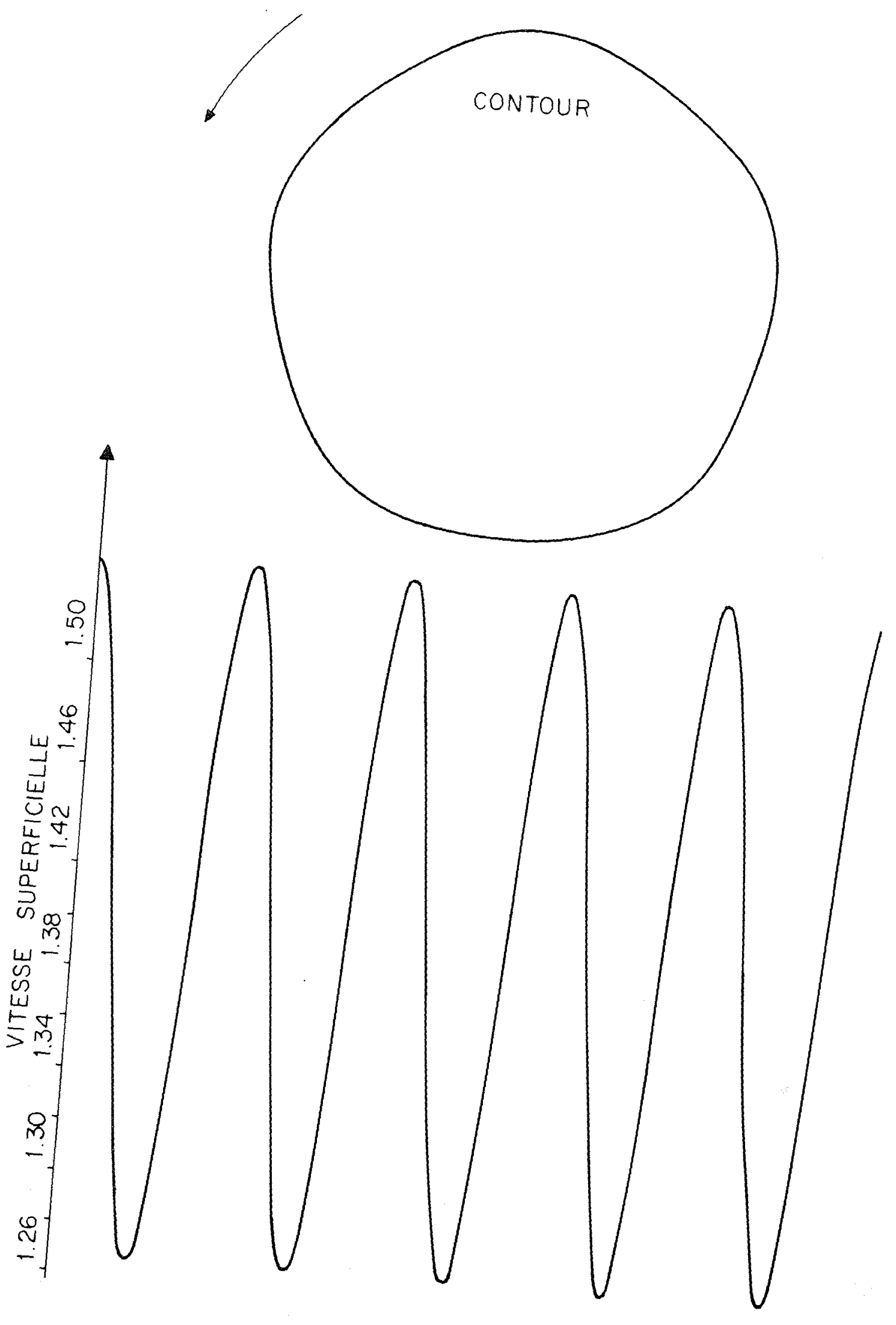

Planche 3/ 
R. GELCl

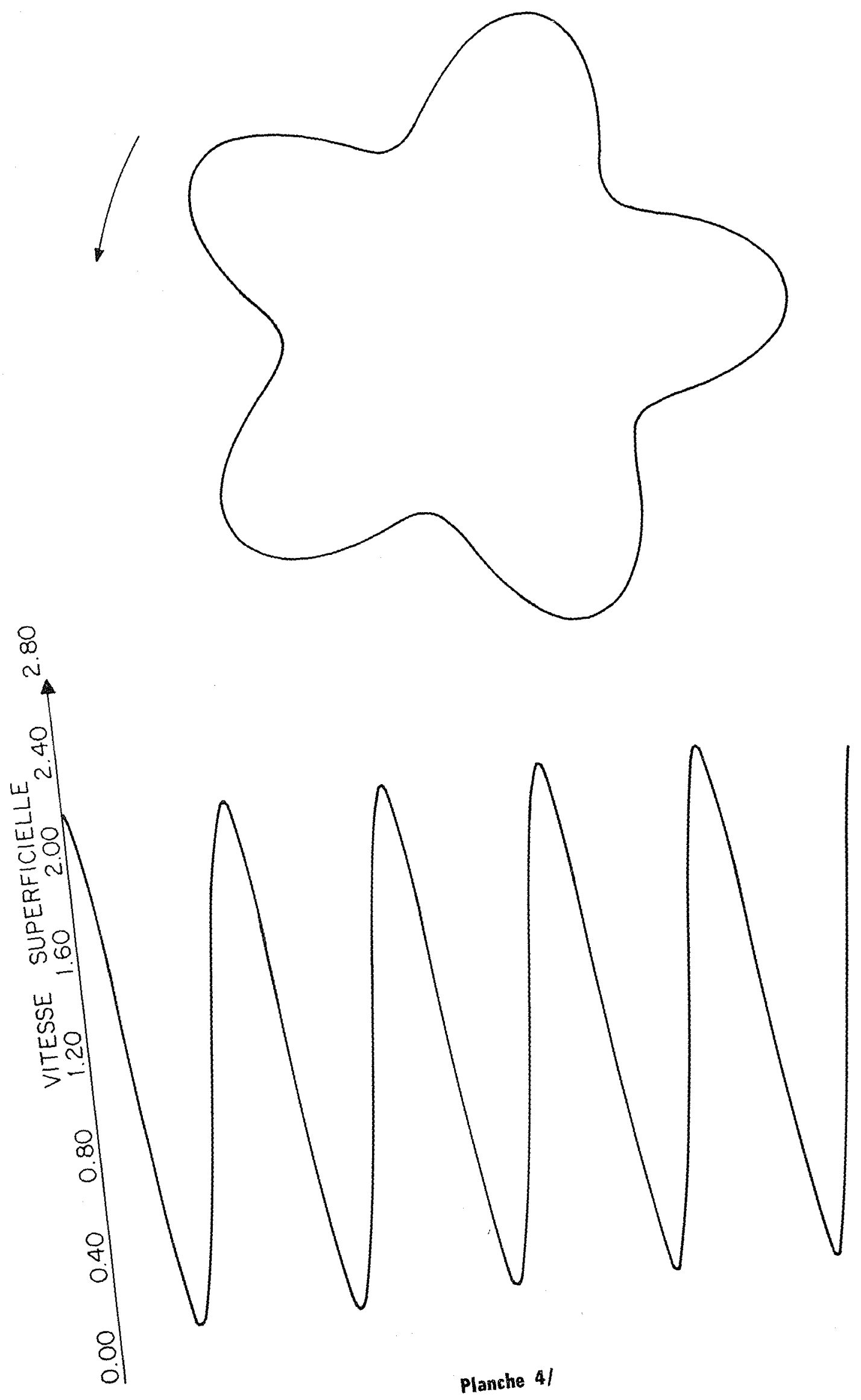

423 


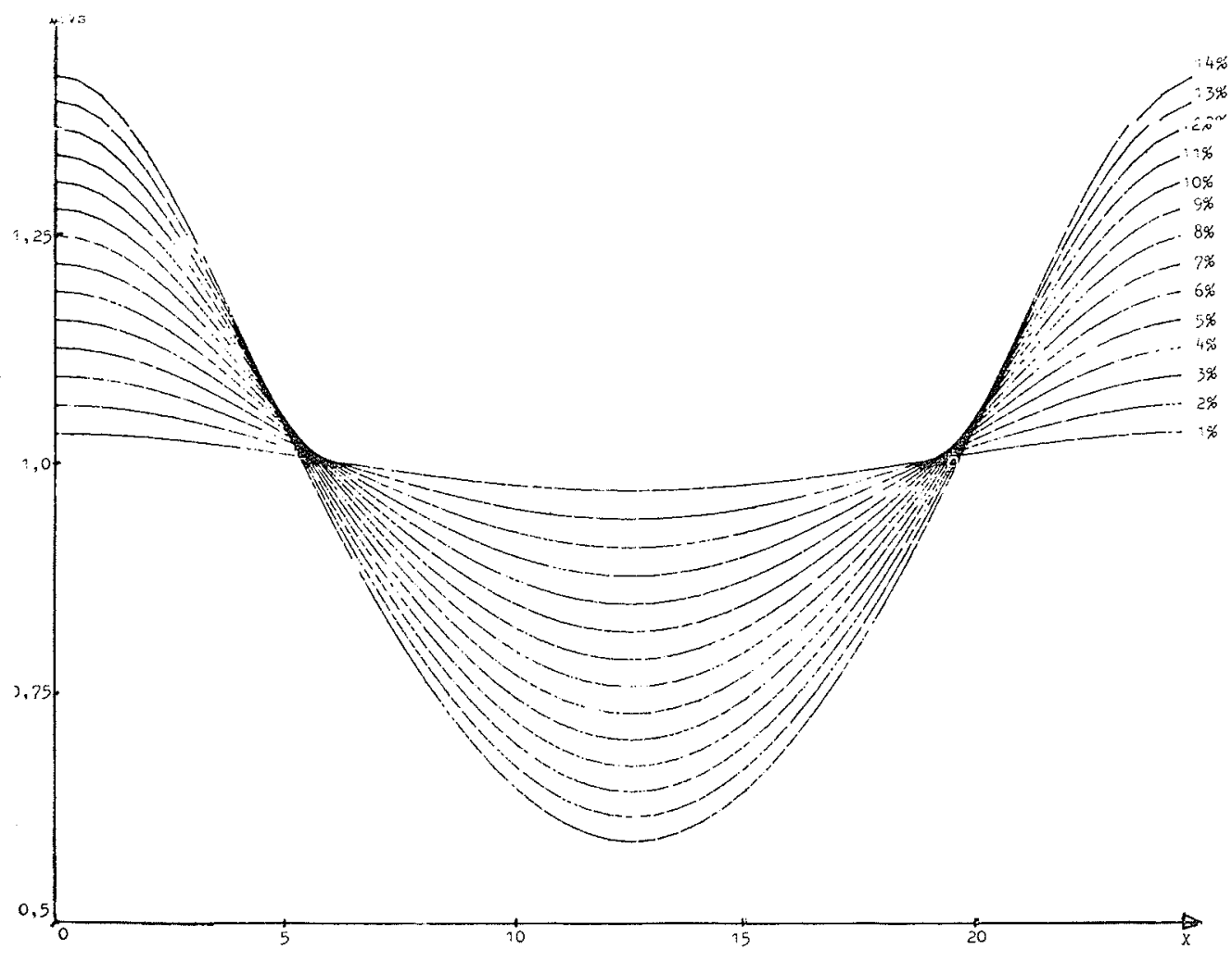

Planche 5/

Profil sinusoidal $L=25 \quad N=50$

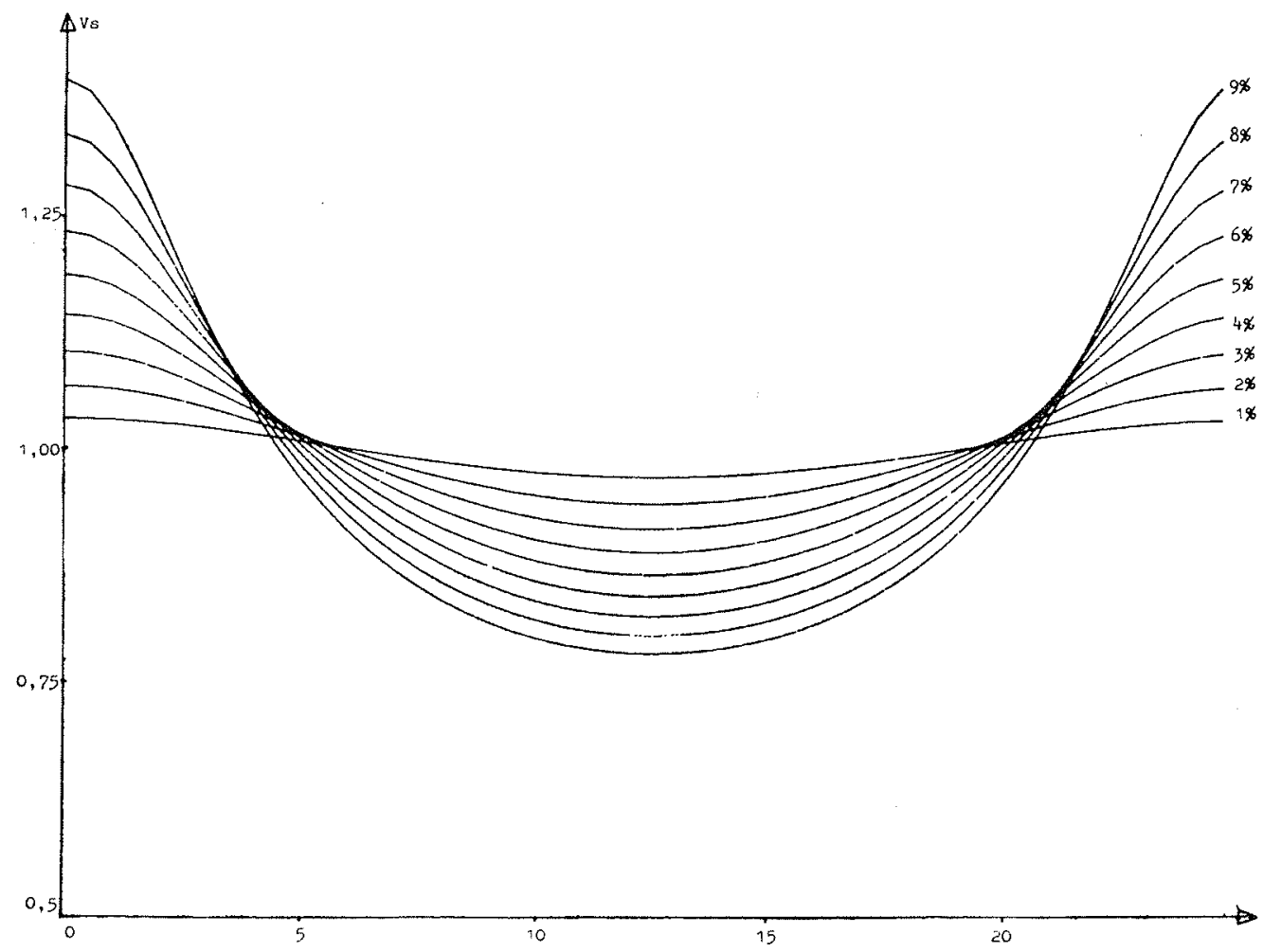

Planche 6/

Profil cycloïdal $\mathrm{L}=25 \quad \mathrm{~N}=50$ 
R. GELCI

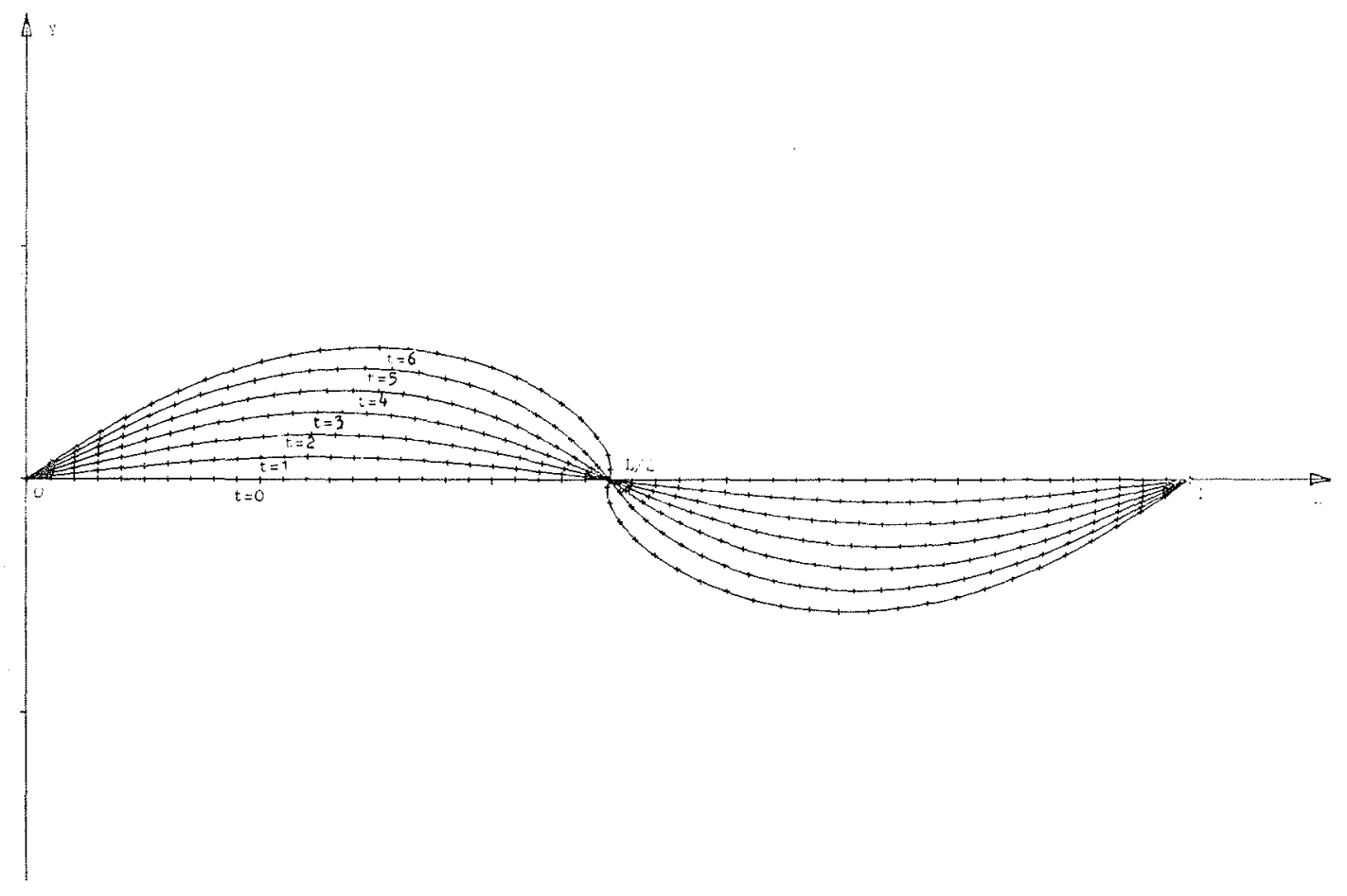

Planche 7 a/

$G(x)=-0,5+0,5 \cos 2 \pi \frac{x}{L}$

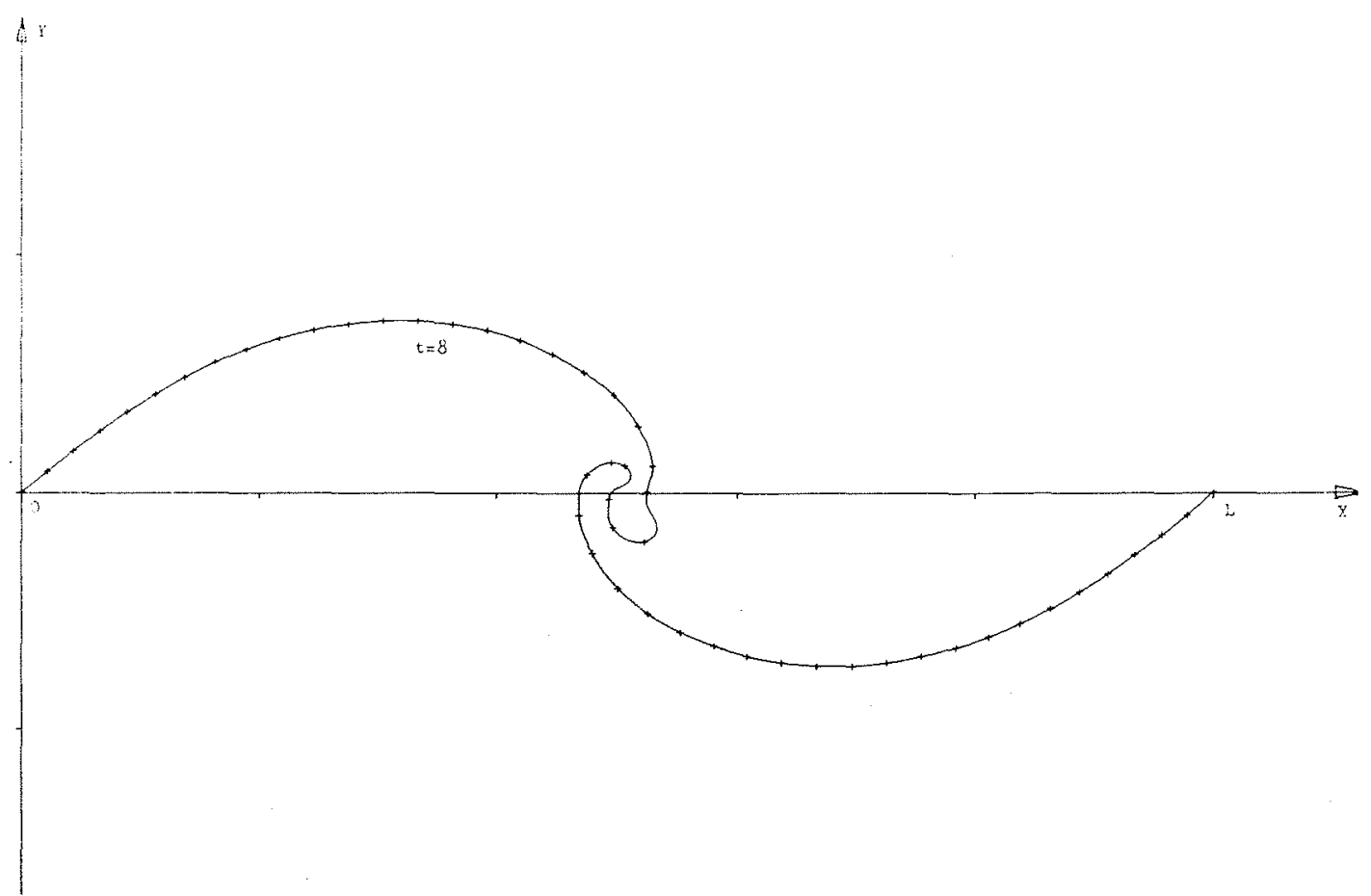

Planche $7 \mathrm{~b} /$ 


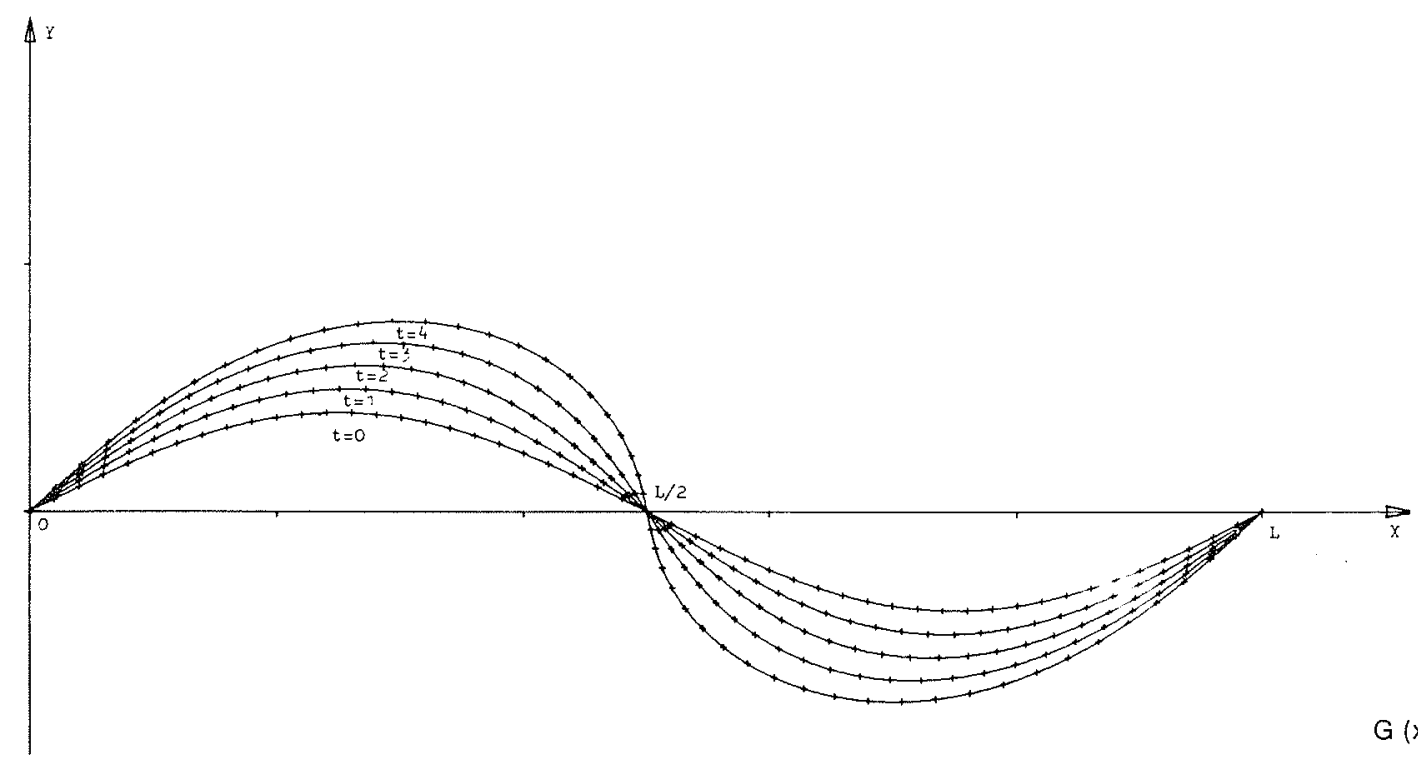

Planche 8 a/

$G(x)=-0,5+0,5 \cos 2 \pi \frac{x}{L}$
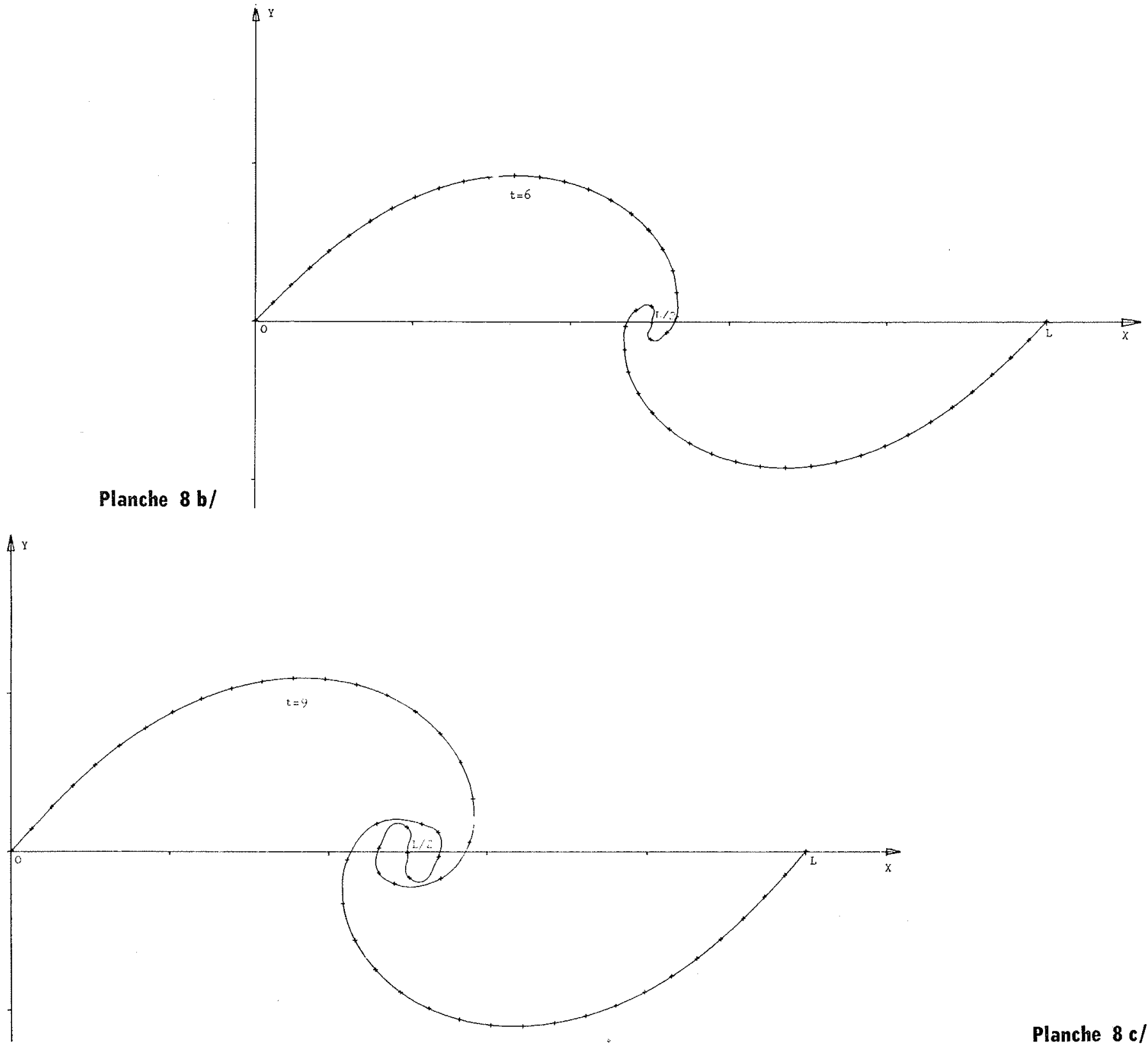


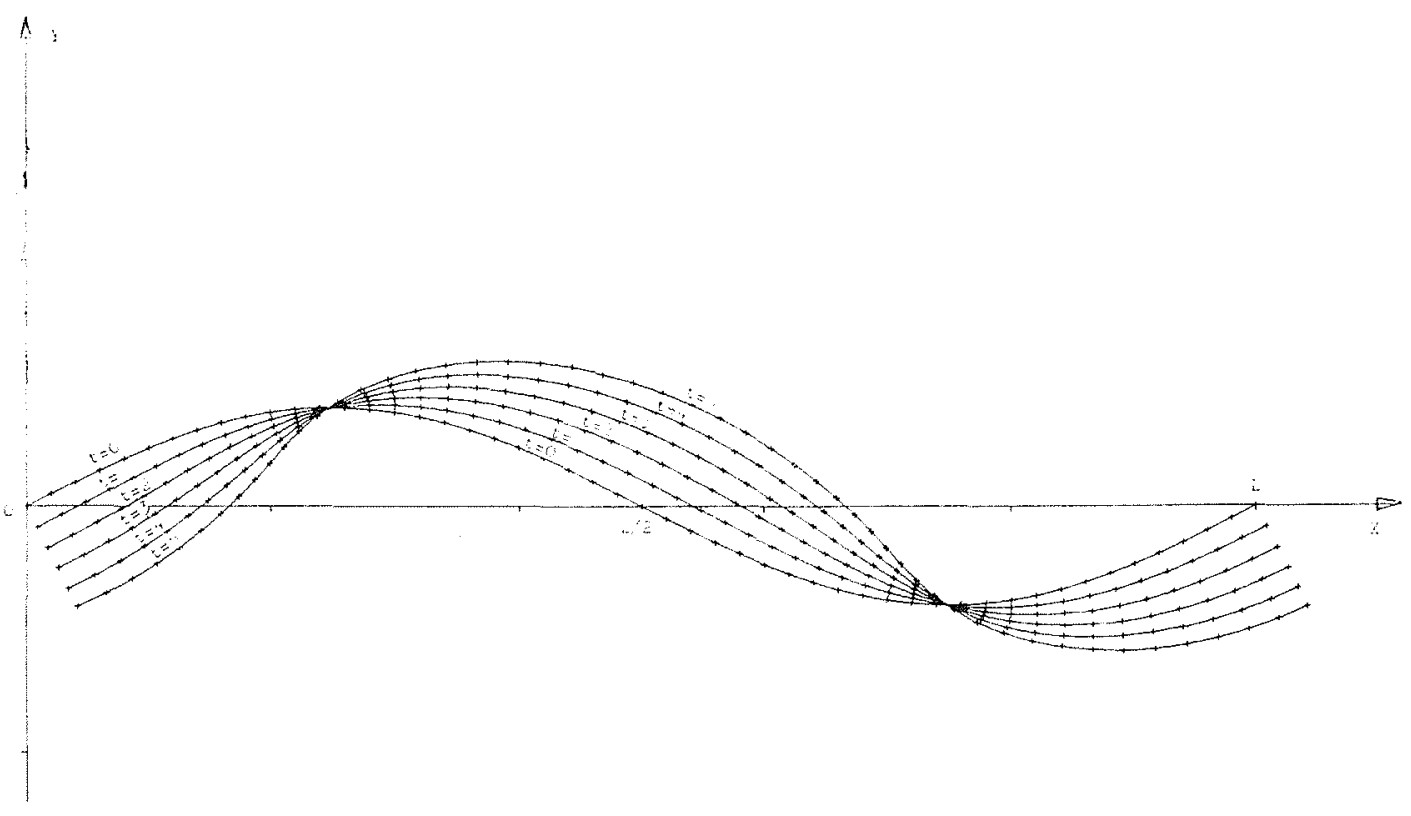

Planche 9 a/

$G(x)=0,5 \sin 2 \pi \frac{x}{L}$

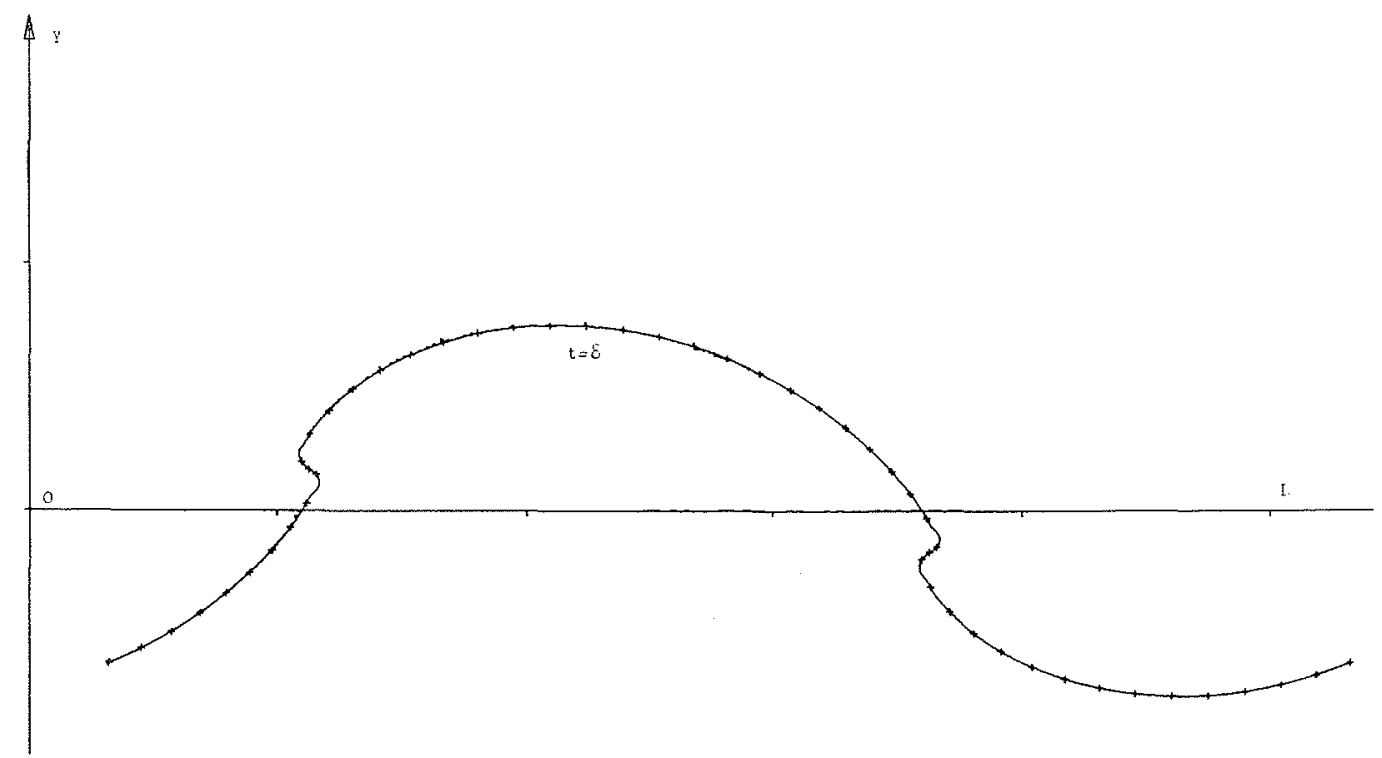

Planche $9 \mathrm{~b}$ / 